\title{
Liebe Mitglieder der Fachgesellschaft,
}

kaum ist unsere Jahrestagung in Fulda Vergangenheit, wirft bereits die nächste Jahrestagung ihren Schatten voraus. Die Vorbereitungen laufen bereits auf Hochtouren. Sie wird am 21. und 22. September 2012 auf Wunsch vieler unserer Mitglieder in Weimar stattfinden. Es wird Sie wieder ein Programm erwarten, das die breite Facette der Reisemedizin abbildet und neben den aktuellen „klassischen“ reisemedizinischen Inhalten auch wieder interessanten fachübergreifenden Themen Raum bietet. Die organisatorische Vorbereitung liegt wie bisher in den Händen unserer Geschäftsstelle, insbesondere hier in den Händen von Ingrid Bergmann, die durch ihren unermüdlichen Einsatz und ihre langjährige Erfahrung zum Erfolg der bisherigen Jahrestagungen viel beigetragen hat. Die inhaltliche Vorbereitung wird dieses Mal unser Vorstandsmitglied Tomas Jelinek übernehmen mit dem Ziel, der mit unserer Umbenennung vom „Verband“ zur „Gesellschaft" verbundenen Aufgabenerweiterung auch thematisch Ausdruck zu verleihen. Wichtiger Aspekt bei der Planung der Jahrestagung wird nach wie vor sein, allen Teilnehmern ein Forum zum Austausch unter reisemedizinischen Experten zu bieten.

Ich lade daher an dieser Stelle auch noch einmal Vorstände und Mitglieder aller anderen in dieser Zeitschrift vertretenen Gesellschaften herzlich dazu ein, zu unserer Jahrestagung zu kommen und dadurch den von unseren Mitgliedern gesuchten „Blick über den Tellerrand“ zu ermöglichen.

In dieser Ausgabe stellen wir ein weiteres, von unserer Gesellschaft herausgegebenes reisemedizinisches Zertifikat vor; dieses Mal „Arbeitsaufenthalt im Ausland unter besonderen klimatischen und ge- sundheitlichen Belastungen“. Nach dem Wegfall der Ermächtigungen für die G-35-Untersuchungen (Tropentauglichkeit) wollten wir damit für Arbeits- und Betriebsmediziner den Anreiz schaffen, sich die für eine Betreuung von ins Ausland entsendeten Arbeitnehmern notwendigen fachlichen Kenntnisse zu verschaffen. In Diskussion ist, ob dieses Zertifikat - losgelöst dann von einer speziellen Weiterbildungsvoraussetzung ein Instrument dafür sein könnte, die Betreuung der jungen Reisenden zu verbessern, die die Bundesregierung im Rahmen ihres Programms „weltwärts“ entsendet.

Weiterhin möchte ich Sie auf die Umfrage zur Fortsetzung unserer reisemedizinischen Exkursionen hinweisen. Wer also Lust hat, zukünftig an einer unserer inzwischen fast schon legendären Exkursionen nach Indien oder Ecuador teilzunehmen, soll sein Interesse unverbindlich durch eine Meldung an unsere Geschäftsstelle bekunden. So können wir den Bedarf abschätzen und weitere reisemedizinische Fortbildungsreisen konkret vorbereiten.

Ich wünsche Ihnen eine besinnliche Adventszeit, frohe Weihnachten und einen guten Rutsch ins neue Jahr 2012.

\section{Mit herzlichen Grüßen}

Ihr Ulrich Klinsing

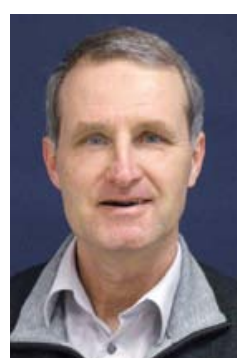

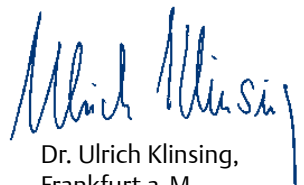
Frankfurt a.M.

\section{DEUTSCHE FACHGESELLSCHAFT

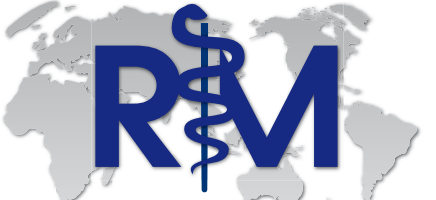 \\ für REISEMEDIZIN e.v. German Society for Travel Medicine}

Hansaallee 299, 40549 Düsseldorf

Tel.: 0211/5202581 Fax: 0211/5202583

E-Mail: info@fachgesellschaft-reisemedizin.de www.fachgesellschaft-reisemedizin.de 


\section{Die DFR-Antwort auf eine arbeitsmedizinische Regelungslücke}

Regelungen haben stets 2 Seiten: Sie fördern das Funktionieren der Gesellschaft, aber um den Preis der Freiheitseinschränkung. Jahrelang mussten Arbeitsmediziner, die die Tauglichkeit von ins Ausland entsandten Mitarbeitern im Rahmen der Untersuchung nach Grundsatz 35 (G 35) beurteilen sollten, die Berechtigung dazu aufwendig erwerben. Mit einem bestandenen Kursus und nachgewiesenen Kenntnissen in der Beurteilung von Arbeitsplätzen in den Tropen und Subtropen konnte man die Ermächtigung beantragen. Das war Qualitätssicherung, auch wenn es oft als Relativierung der Kompetenz erlebt wurde, die man sich durch die Weiterbildung erworben hatte.

Die zum 01.01.2009 in Kraft getretene Verordnung zur arbeitsmedizinischen Vorsorge (ArbMedVV) räumte mit diesem Verfahren überraschend auf. Nun waren plötzlich alle Arbeitsmediziner im Grundsatz berechtigt, alle Vorsorgeuntersuchungen einschließlich der nach G 35 durchzuführen. Aus der Ermächtigung wurde eine wesentlich unverbindlichere im $\S 7$ niedergelegte Formulierung, nach der ein Arzt, der über die „erforderlichen Fachkenntnisse oder die speziellen Anerkennungen“ nicht verfügt, einen Kollegen hinzuziehen muss. Die formalisierten Voraussetzungen aber waren ja gerade entfallen. War denn mehr als der Facharzt „erforderlich“? Und auch andersherum kamen Kollegen, die bislang mit Arbeitsplätzen im Ausland nichts zu tun hatten, unter Druck, von dort zurückgekehrte Mitarbeiter auf Zeichen einer Berufskrankheit hin zu untersuchen, da die Firma dies von ihnen verlangte.

\section{Klare Position der DFR}

Die DFR hat sich dazu bereits frühzeitig klar positioniert. Zusätzlich zur arbeitsmedizinischen Kompetenz ist eine reisemedizinische Qualifikation erforderlich, deren Zuschnitt sich an den zu erwartenden Fragestellungen orientiert:

- Ist ein Arbeitnehmer für einen Auslandsposten geeignet?

- Wie wirkt sich eine Vorerkrankung dort aus?

- Von welcher Infrastruktur kann oder muss ich ausgehen?

- Wenn im Rahmen der Nachuntersuchung Krankheitszeichen angegeben werden - woran ist zu denken?

Für die Abbildung dieser Fragestellung kam uns das modulare Konzept sehr entgegen, mit dem wir die Inhalte der

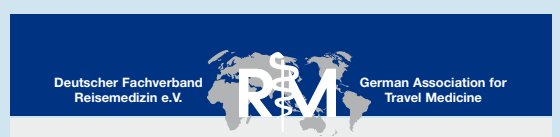
Reisemedizin in einzelne inhaltliche Blöcke zusammengefasst haben. Daher wurde der Beschluss gefasst, die Kombination aus

- Basiszertifikat Reisemedizin (32-h-Kurs),

- Aufbaumodul 6 „Internationale Arbeitseinsätze u. Langzeitaufenthalte“ ( 8 h) und

- Aufbaumodul 12 „Gesundheitsstörungen bei Reiserückkehrern“

als separate Qualifikation unter dem Namen „Arbeitsaufenthalt im Ausland unter besonderen klimatischen und gesundheitlichen Belastungen“ anzubieten. Die inhaltliche Ausrichtung ist über unsere neue Homepage, www.fachgesellschaftreisemedizin.de, für jeden, auch jeden potenziellen Kursveranstalter, abrufbar.

Die DFR veranstaltet ja keine eigenen Kurse, sondern normiert und zertifiziert - dies ist eine bewusste Vermeidung des Konflikts zwischen inhaltlicher und wirtschaftlicher Arbeit, die sich sehr bewährt hat. Inzwischen haben die ersten Kollegen dieses Zertifikat erhalten - ein wichtiger Schritt zu besserer Vorsorge für Auslandsmitarbeiter, aber auch im Konfliktfall eine Argumentationshilfe, wenn Kollegen die „erforderlichen Fachkenntnisse“ nachweisen müssen.

Dr. Burkhard Rieke, Düsseldorf 


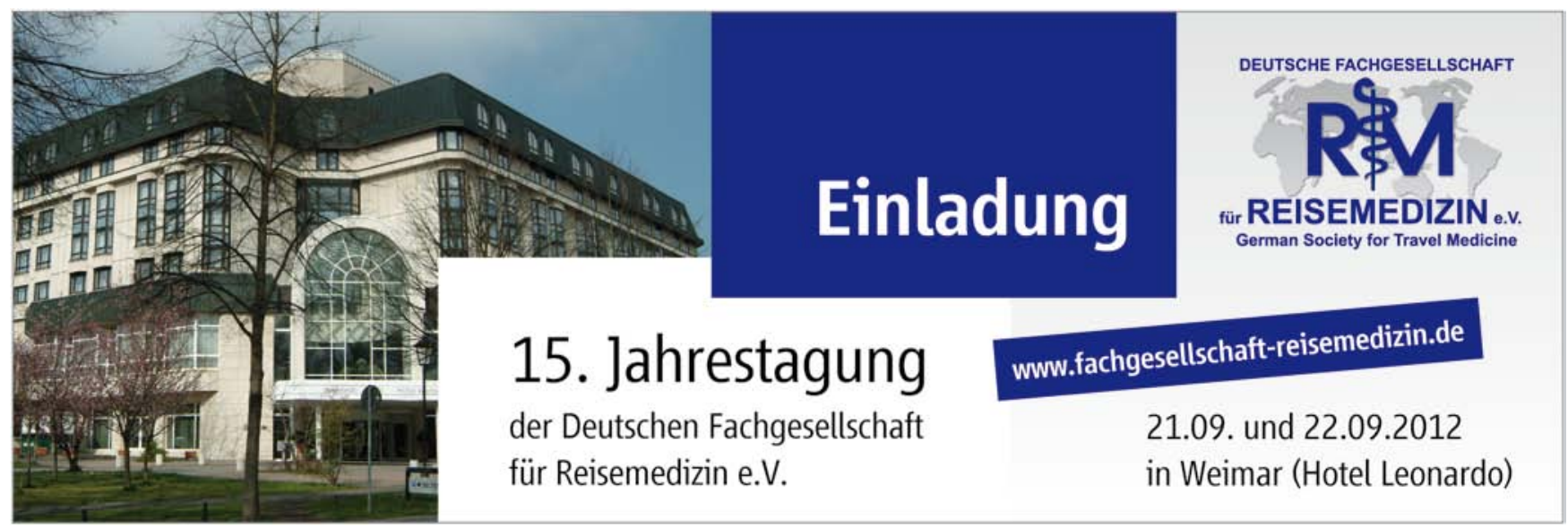

Wir möchten Sie herzlich einladen, an unserer nächsten Jahrestagung, im September 2012, teilzunehmen. Tagungsort ist diesmal das Leonardo Hotel in der Kulturstadt Weimar (Belvederer Allee 25, 99425 Weimar).

Das Programm der 15. Jahrestagung wird sich u. a. aus folgenden Themen zusammensetzen:

- Reisemedizinische Fallbeispiele

- Fallbeispiele aus der Rückkehrermedizin

- DD des Fiebers beim Tropenrückkehrer

- Malaria: aktuelle Trends

- Impfungen: aktuelle Trends

- Impfen bei Immunsuppression

- Wilderness Medicine

- Aktuelles aus der höhenmedizinischen Forschung

- Tuberkulosediagnostik beim beruflichen Auslandsaufenthalt und beim Tropenrückkehrer

- Reisemedizin im europäischen Vergleich: Ausbildungsstrukturen und Beratungsstandards

Die Zertifizierung der Veranstaltung wird bei der zuständigen Landesärztekammer beantragt.

Weitere Informationen finden Sie auf unserer Homepage www.fachgesellschaft-reisemedizin.de.

Eine Kopiervorlage zur Anmeldung finden Sie hier.

Anmeldeformular

(bitte per Fax an 0211/5202583 oder an u.g. Postanschrift)

Ich nehme teil am:

$\square$ Freitag, 21.09.2012

Samstag, 22.09.2012

Tagungsgebühr für einen einzelnen Tag:

Mitglieder $90 €$ - Nichtmitglieder $110 €$

Tagungsgebühr für beide Tage:

Mitglieder $160 €$ - Nichtmitglieder $200 €$

(In den Tagungsgebühren sind Tagungsgetränke, Kaffeepausen, Stadtrundgang und Abendessen am Freitag (exkl. Getränke) sowie Mittagessen am Samstag enthalten.)

Zahlungsweise:

$\square$ Die Tagungsgebühr werde ich nach Erhalt der Rechnung auf das Konto der Deutschen Fachgesellschaft für Reisemedizin e.V. überweisen

Die Tagungsgebühr soll nach Rechnungsstellung von meinem Bank-Konto abgebucht werden
Name, Vorname:

Institution:

Straße:

PLZ/Ort:

Telefon: Fax:

DFR-Mitgliedsnummer (wenn vorhanden):

Bankverbindung (nur bei Abbuchungsauftrag):

Kto-Nr:

BLZ:

Bank:

Datum:

Unterschrift: 